\title{
Caffeic acid phenethyl ester reduces the secretion of vascular endothelial growth factor through the inhibition of the ROS, PI3K and HIF-1 $\alpha$ signaling pathways in human retinal pigment epithelial cells under hypoxic conditions
}

\author{
SUNG HWA PAENG ${ }^{1 *}$, WON-KYO JUNG ${ }^{2 *}$, WON SUN PARK ${ }^{3 *}$, DAE-SUNG LEE ${ }^{4}$, \\ GI-YOUNG KIM ${ }^{5}$, YUNG HYUN CHOI ${ }^{6}$, SU-KIL SEO ${ }^{7}$, WON HEE JANG ${ }^{8}$, JUNG SIK CHOI ${ }^{9}$, \\ YOUNG-MIN LEE ${ }^{9}$, SAEGWANG PARK $^{7}$ and IL-WHAN CHOI ${ }^{7}$ \\ ${ }^{1}$ Department of Neurosurgery, Busan Paik Hospital, Inje University College of Medicine; \\ ${ }^{2}$ Department of Biomedical Engineering, and Center for Marine-Integrated Biomedical Technology (BK21 Plus), \\ Pukyong National University, Busan; ${ }^{3}$ Department of Physiology, Kangwon National University School of Medicine, \\ Chuncheon, Gangwon; ${ }^{4}$ Marine Biodiversity Institute of Korea, Seocheon, Chungcheongnam-do; \\ ${ }^{5}$ Laboratory of Immunobiology, Department of Marine Life Sciences, Jeju National University, Jeju; \\ ${ }^{6}$ Department of Biochemistry, College of Oriental Medicine, Dongeui University; Departments of ${ }^{7}$ Microbiology, \\ ${ }^{8}$ Biochemistry, and ${ }^{9}$ Internal Medicine, Busan Paik Hospital, Inje University College of Medicine, Busan, Republic of Korea
}

Received October 14, 2014; Accepted February 24, 2015

DOI: $10.3892 / \mathrm{ijmm} .2015 .2116$

\begin{abstract}
Choroidal neovascularization (CNV) can lead to progressive and severe visual loss. Vascular endothelial growth factor (VEGF) promotes the development of CNV. Caffeic acid phenethyl ester (CAPE), a biologically active component of the honeybee (Apis mellifera) propolis, has been demonstrated to have several interesting biological regulatory properties. The objective of this study was to determine whether treatment with CAPE results in the inhibition of the production of vascular endothelial growth factor (VEGF) in retinal pigment epithelial cells (RPE cells) under hypoxic conditions and to explore the possible underlying mechanisms. An in vitro experimental model of hypoxia was used to mimic an ischemic microenvironment
\end{abstract}

Correspondence to: Professor Il-Whan Choi or Professor Saegwang Park, Department of Microbiology, Inje University College of Medicine, 75 Bokji-Ro, Busanjin-gu, Busan 614-735, Republic of Korea

E-mail: cihima@inje.ac.kr

E-mail:micpsg@inje.ac.kr.ac.kr

*Contributed equally

Abbreviations: AMD, age-related macular degeneration; CNV, choroidal neovascularization; VEGF, vascular endothelial growth factor; CAPE, caffeic acid phenethyl ester; RPE, retinal pigment epithelium; HIF-1 $\alpha$, hypoxia-inducible factor-1 $\alpha$; ROS, reactive oxygen species

Key words: hypoxia, caffeic acid phenethyl ester, human retinal pigment epithelium, choroidal neovascularization, vascular endothelial growth factor for the RPE cells. Human RPE cells (ARPE-19) were exposed to hypoxia with or without CAPE pre-treatment. ARPE-19 cells were used to investigate the pathway involved in the regulation of VEGF production under hypoxic conditions, based on western blot analysis, enzyme-linked immunosorbent assay (ELISA) and electrophoretic mobility shift assay (EMSA). The amount of VEGF released from the hypoxia-exposed cells was significantly higher than that of the normoxic controls. Pre-treatment with CAPE suppressed the hypoxia-induced production of VEGF in the ARPE-19 cells, and this effect was inhibited through the attenuation of reactive oxygen species (ROS) production, and the inhibition of phosphoinositide 3-kinase (PI3K)/AKT and hypoxia-inducible factor-1 $\alpha$ (HIF-1 $\alpha)$ expression. These in vitro findings suggest that CAPE may prove to be a novel anti-angiogenic agent for the treatment of diseases associated with CNV.

\section{Introduction}

Age-related macular degeneration (AMD) is the leading cause of permanent blindness in older adults. The majority of patients with AMD experience severe vision loss in the center of the macula due to damage to the retina. There are two primary types of AMD, 'wet' and 'dry'. 'Dry' AMD is characterized by late-stage geographic atrophy resulting from the gradual degeneration of retinal cells, and 'wet' AMD is caused by choroidal neovascularization (CNV) (1). 'Wet' AMD causes more severe visual loss, but is more treatable. $\mathrm{CNV}$ is a common sign of the wet form of AMD, which is also referred to as the exudative form of AMD (2). CNV sprouts from choroidal vessels and extends through Bruch's membrane and the retinal pigment epithelium (RPE) to reach the subretinal space (3). The RPE, which lies between the photoreceptor cell layer of 
the retina and Bruch's membrane and choroid, is a monolayer of pigmented cells (4). It plays a major role in retinal physiology by supporting the function of the photoreceptors and pathology in a variety of retinal diseases (5).

Retinal hypoxia (oxygen deficiency), which occurs with capillary non-perfusion and leads to angiogenesis, is a major pathological condition underlying a number of sight-threatening diseases, including diabetic retinopathy (DR), retinopathy of prematurity (ROP), AMD and neovascular glaucoma (6-8). Hypoxia occurs secondary to a number of disease processes in the human body (9). In response to hypoxia, retinal pigment epithelial cells (RPE cells) rapidly release various growth factors resulting in angiogenesis, fibrovascular tissue formation and retinal ablation (10). Among the hypoxia-stimulated growth factors, vascular endothelial growth factor (VEGF), a potent vascular endothelial cell mitogen, is a pivotal regulator of vasculogenesis and angiogenesis in CNV (11). It has been demonstrated that the overproduction of VEGF by RPE cells induces CNV (12), and VEGF antagonists have been shown to attenuate $\mathrm{CNV}$ in animal models $(13,14)$. Therefore, therapeutic agents that inhibit the overproduction of VEGF have shown promising inhibitory effects on CNV in patients with AMD.

Caffeic acid phenethyl ester (CAPE), a potent flavonoid antioxidant, is the active component of honeybee (Apis mellifera) propolis. CAPE has profound antiviral, antitumoral, anti-inflammatory, antioxidant, neuroprotective, anti-atherosclerotic and immunomodulatory properties in diverse systems (15). CAPE has also been reported to have anti-angiogenic activity in cancer $(16,17)$. However, to the best of our knowledge, there is no study available to date evaluating the effects of CAPE on VEGF expression in CNV-associated angiogenesis. Previous studies have indicated that oxidative stress, inflammatory responses and atherosclerotic properties are associated with the pathogenesis of CNV in patients with AMD (18-20). Therefore, the antioxidant effects of CAPE may be associated with a reduced incidence of $\mathrm{CNV}$. However, there is no experimental evidence to support this suggestion. In the present study, we investigated whether treatment with CAPE results in the inhibition of VEGF production and the possible mechanisms involved in these effects in ARPE-19 cells under hypoxic conditions.

\section{Materials and methods}

Reagents. CAPE, U0126 and diphenyleneiodonium (DPI) were purchased from the Sigma Chemical Co. (St. Louis, MO, USA). SB203580 was purchased from Enzo Life Sciences, Inc. (Farmingdale, NY, USA). LY294002 was purchased from Calbiochem (San Diego, CA, USA). YCG063 [an inhibitor of mitochondrial reactive oxygen species (ROS)] was obtained from Millipore (Billerica, MA, USA). Dulbecco's modified Eagle's medium/nutrient mixture F12 (DMEM/F12 medium), fetal bovine serum (FBS) and trypsin-EDTA were obtained from Invitrogen-Gibco (Carlsbad, CA, USA). Antibody against hypoxia-inducible factor-1 $\alpha$ (HIF-1 $\alpha$; NB100-105) was obtained from Novus Biologicals (Littleton, CO, USA). Antibody against histone H3 (SC-10809) was purchased from Santa Cruz Biotechnology, Inc. (Santa Cruz, CA, USA). Antibodies against AKT (\#9272), phosphorylated (p)-AKT (ser473; \#4058) and p-PI3K (\#4255) were purchased from Cell Signaling Technology (Beverly, MA, USA). Nitrocellulose membranes and an enhanced chemiluminescence (ECL) kit were obtained from Amersham Pharmacia Biotech (Uppsala, Sweden).

Cell culture. The human RPE cell line (ARPE-19) was obtained from the American Type Culture Collection (ATCC, Manassas, VA, USA) and cultured in DMEM/F12 medium supplemented with $10 \%$ FBS plus $100 \mathrm{IU} / \mathrm{ml}$ penicillin and $100 \mu \mathrm{g} / \mathrm{ml}$ streptomycin mixture (Gibco/BRL, Gaithersburg, MD, USA) in a $5 \% \mathrm{CO}_{2}$ humidified atmosphere at $37^{\circ} \mathrm{C}$. The ARPE-19 cells were trypsinized, seeded in $10-\mathrm{cm}$ diameter dishes, and incubated overnight until attachment.

Induction of hypoxia. Following overnight incubation, the cells were moved to a hypoxic chamber (Galaxy 14S; Eppendorf, Enfield, CT, USA). Before the cells were exposed to hypoxia, the medium was replaced with DMEM/F12 medium. The hypoxic chamber was equipped with an oxygen sensor and gas regulator and was flushed with $1 \% \mathrm{O}_{2}, 5 \% \mathrm{CO}_{2}$ and $94 \% \mathrm{~N}_{2}$, sealed, and placed at $37^{\circ} \mathrm{C}$. After reaching $\sim 80 \%$ confluency, the cells were subjected to hypoxic conditions in the hypoxic chamber for $24 \mathrm{~h}$. The cells cultured under hypoxic conditions were processed in the chamber itself to avoid any exposure to normoxic conditions.

Cell viability assay. The viability of the ARPE-19 cells was determined using the cell counting kit-8 (CCK-8) according to the manufacturer's instructions (Dojindo Laboratories, Kumamoto, Japan). Briefly, the cells were seeded in triplicate at a density of $1 \times 10^{4}$ cells/well in 96 -well culture plates and allowed to attach overnight. The medium was then replaced with $100 \mu \mathrm{l}$ DMEM/F12 medium containing 0, 10, 20 and $40 \mu \mathrm{M}$ of CAPE. The plates were incubated for $24 \mathrm{~h}$ under hypoxic conditions, and $10 \mu \mathrm{l}$ of $\mathrm{CCK}-8$ reagent was added to each well. After another $2 \mathrm{~h}$ incubation at $37^{\circ} \mathrm{C}$, the plates were read at $450 \mathrm{~nm}$ using a microplate reader (Model EL800; Bio-Tek Instruments, Inc., Winooski, VT, USA).

Enzyme-linked immunosorbent assay (ELISA). The VEGF levels in the cell culture medium were assessed by ELISA. The cells were treated with various concentrations of CAPE for $2 \mathrm{~h}$ before being exposed to hypoxic conditions. Follwoing incubation for $24 \mathrm{~h}$ under hypoxic conditions, the culture supernatant was collected and the VEGF levels were measured using a VEGF DuoSet ELISA Development kit (R\&D Systems, Minneapolis, MN, USA) according to the manufacturer's instructions. The absorbance at $450 \mathrm{~nm}$ was determined using a microplate reader (Model EL800; Bio-Tek Instruments, Inc.).

Western blot analysis. Western blot analysis was performed as previously described (21). The ARPE-19 cells were washed 3 times with phosphate-buffered saline (PBS) and lysed with lysis buffer (Mammalian Cell-PE LB; G-Biosciences, St. Louis, MO, USA). Equal amounts of protein were separated on $10 \%$ SDS-polyacrylamide minigels and transferred onto nitrocellulose transfer membranes. Following incubation with the appropriate primary antibody, the membranes were incubated for $1 \mathrm{~h}$ at room temperature with a secondary antibody conjugated to horseradish peroxidase. Following 3 washes in Tris-Buffered Saline and Tween-20 (TBST), the immunoreactive bands were visualized using the ECL detection system (Pierce, Rockford, IL, USA). 
Preparation of nuclear extracts and electrophoretic mobility shift assay (EMSA). Nuclear extracts were prepared using the NE-PER nuclear extraction reagent (Pierce). For the gel retardation assay, a typical double-stranded oligonucleotide for the HIF-1 $\alpha$ binding DNA sequence (5'-TCTGTACGTGA CCACACTCACCTC-3' with the HIF-1 $\alpha$ binding site sequence underlined) was purchased from Santa Cruz Biotechnology, Inc. (Cat. no. sc-2625). A non-radioactive method in which the 3 ' end of the probe was labeled with biotin was used in these experiments (Pierce). The binding reactions contained $5 \mu \mathrm{g}$ of nuclear extract protein, buffer $(10 \mathrm{mM}$ Tris, $\mathrm{pH}$ 7.5, $50 \mathrm{mM}$ $\mathrm{KCl}, 5 \mathrm{mM} \mathrm{MgCl}{ }_{2}, 1 \mathrm{mM}$ dithiothreitol, $0.05 \%$ Nonidet P-40, and $2.5 \%$ glycerol $), 50 \mathrm{ng}$ of poly(dI-dC) and $20 \mathrm{fM}$ of the biotin-labeled DNA. The reactions were incubated for $20 \mathrm{~min}$ at room temperature in a final volume of $20 \mu \mathrm{l}$. The competition reactions were conducted by the addition of a 25 -fold excess of unlabeled HIF-1 $\alpha$ to the reaction mixture. The mixture was then separated by electrophoresis on a $5 \%$ polyacrylamide gel in $0.5 \mathrm{X}$ Tris-borate buffer and transferred onto nylon membranes. The biotin-labeled DNA was detected using a LightShift Chemiluminescent EMSA kit (Pierce).

Assay of intracellular ROS levels. Intracellular ROS levels were measured using the 2',7'-dichlorofluorescein diacetate (DCF-DA) assay as previously described with some modifications (22). DCF-DA can be deacetylated in cells, where it reacts quantitatively with intracellular radicals to convert into its fluorescent product, DCF, which is retained within the cells. Therefore, DCF-DA is used to evaluate the generation of ROS under conditions of oxidative stress. The ARPE-19 cells $\left(1 \times 10^{4}\right.$ cells/well) were seeded in 96 -well plates in a humidified atmosphere containing $5 \% \mathrm{CO}_{2}$ at $37^{\circ} \mathrm{C}$ for $16 \mathrm{~h}$. Following $16 \mathrm{~h}$ of incubation, the cells were treated with various concentrations (10 and $20 \mu \mathrm{M})$ of CAPE and further incubated for $24 \mathrm{~h}$ under hypoxic conditions. Thereafter, the cells were incubated with $10 \mu \mathrm{M}$ DCF-DA for 30 min under hypoxia conditions. Subsequently, the cells were fixed with an equal volume of $4 \%$ formaldehyde and were analyzed immediately after that. The intracellular ROS levels were measured using a fluorescent plate reader (SpectraMax M2; Molecular Devices, Sunnyvale, CA, USA) at an excitation wavelength of $492 \mathrm{~nm}$ and an emission wavelength of $515 \mathrm{~nm}$.

Statistical analysis. Data values represent the means \pm SD. To analyze the data produced from the experiments with two independent variables, a one-way analysis of variance (ANOVA) was performed using GraphPad Prism software (GraphPad Software, La Jolla, CA, USA). A value of $\mathrm{p}<0.05$ was considered to indicate a statistically significant difference.

\section{Results}

Production of VEGF under hypoxic conditions. Initially, we wished to evaluate whether we could use our system to induce the hypoxia signaling pathway by measuring the production of VEGF. One of the main characteristics of CNV is the formation of new blood vessels; this may be associated with the VEGF levels as VEGF is the main inducer of neoangiogenesis (23). Based on ELISA, the ARPE-19 cells were exposed to normoxic and hypoxic conditions for 2, 4, 8, 16, 24 and $48 \mathrm{~h}$ (Fig. 1A).
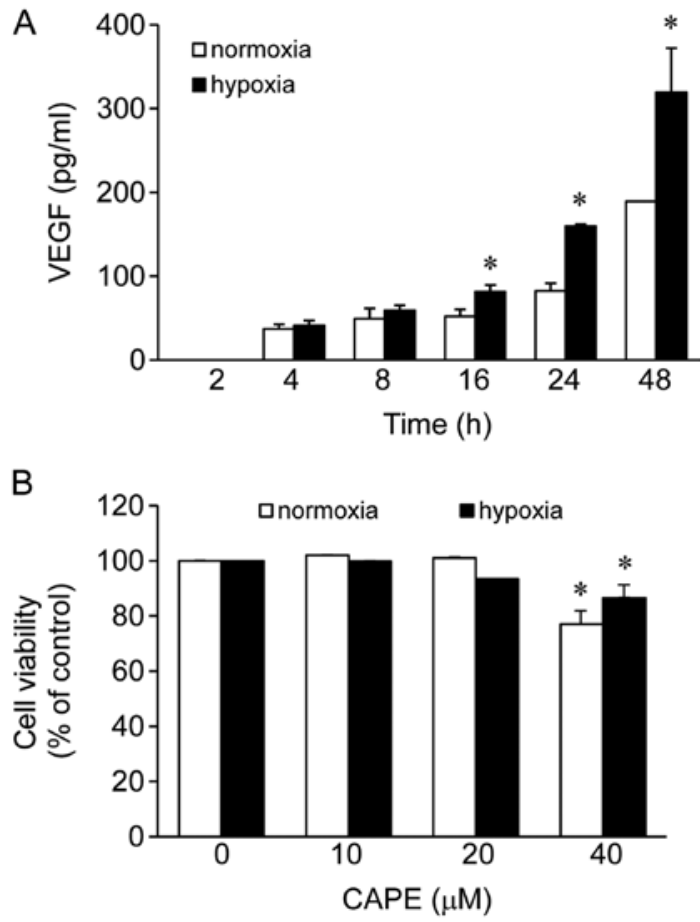

Figure 1. Expression of (A) vascular endothelial growth factor (VEGF) and (B) viability of ARPE-19 cells following exposure to hypoxic and normoxic conditions. (A) Cells were exposed to hypoxic or normoxic conditions for the indicated periods of time (2, 4, 8, 16, 24 and $48 \mathrm{~h})$. VEGF levels were measured in the culture medium using a commercial ELISA kit. ${ }^{*} \mathrm{p}<0.05$ vs. same time point under normoxic conditions. (B) Cells were treated with the indicated concentrations of caffeic acid phenethyl ester (CAPE) $(0,10,20$ or $40 \mu \mathrm{M}$ ) for $24 \mathrm{~h}$ prior to exposure to hypoxia or normoxia for $2 \mathrm{~h}$, and cell viability was determined. Cell viability was assessed using the cell counting kit-8 (CCK-8). The results are expressed as the percentage of surviving cells over the control cells (no addition of CAPE under hypoxic or normoxic conditions). Each value indicates the mean $\pm \mathrm{SD}$ and is representative of results obtained from 3 independent experiments.

The production of VEGF was elevated following the exposure of the cells to both hypoxic and normoxic conditions as the exposure time increased (Fig. 1A). The production of VEGF was not significantly altered following the exposure of the cells to hypoxic conditions for 4 and $8 \mathrm{~h}$ as indicated by the 1.1- and 1.2-fold-change in its levels, respectively, compared to exposure to normoxic conditions (Fig. 1A). At the 2-h time point, VEGF was not produced under either normoxic or hypoxic conditions. However, the levels of VEGF were significantly elevated following the exposure of the cells to hypoxic conditions for 16,24 and $48 \mathrm{~h}$ as indicated by the 1.6- (change from 52.2 to 81.34), 1.9- (change from 82.45 to 159.6) and 1.7- (change from 189.2 to 319.52 ) fold change, respectively, compared to exposure to normoxic conditions (Fig. 1A). Among these 3 time points, compared to the levels observed following exposure to normoxic conditions for $24 \mathrm{~h}$, exposure to hypoxic conditions for $24 \mathrm{~h}$ demonstrated the maximum elevation in the VEGF levels (Fig. 1A). Therefore, we selected this time point for all the subsequent experiments.

Effects of CAPE on the viability of human ARPE-19 cells. We then examined the viability of human ARPE-19 cells treated with CAPE $(10,20$ and $40 \mu \mathrm{M})$ using the CCK-8 assay under normoxic or hypoxic conditions. No cytotoxic effect on the 


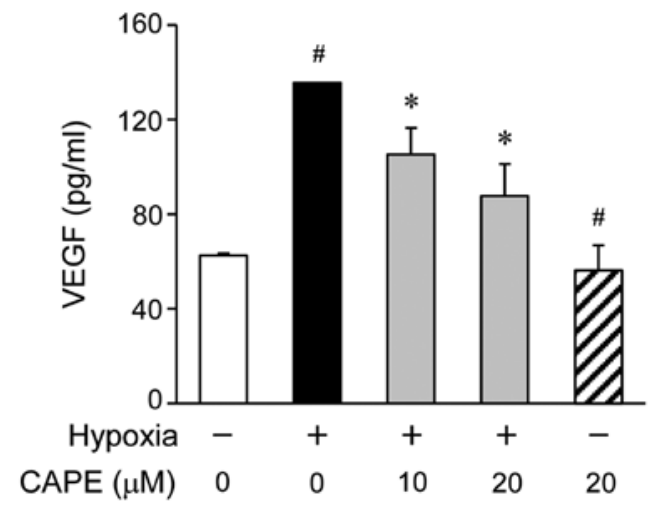

Figure 2. Effect of caffeic acid phenethyl ester (CAPE) on the production of vascular endothelial growth factor (VEGF) in ARPE-19 cells following exposure to hypoxic conditions $(24 \mathrm{~h})$. The levels of VEGF in the culture medium were determined by ELISA. The cells were treated with CAPE $(10$ or $20 \mu \mathrm{M})$ for $2 \mathrm{~h}$ prior to exposure to hypoxic conditions for $24 \mathrm{~h}$. Each bar represents the mean \pm SD from 3 independent experiments. " $\mathrm{p}<0.05$ vs. normoxia; p $\mathrm{p}<0.05$ vs. untreated group under hypoxic conditions.

human ARPE-19 cells was observed at doses of up to $20 \mu \mathrm{M}$, but cell viability was reduced by 23 and $13.5 \%$ with the $40 \mu \mathrm{M}$ dose of CAPE (Fig. 1B) under normoxic and hypoxic conditions, respectively. Based on these results, a concentration of $10-20 \mu \mathrm{M}$ of CAPE was selected for the subsequent experiments.

Effect of CAPE on VEGF production under hypoxic conditions. To examine the inhibitory effects of CAPE on the hypoxia-induced production of VEGF in the ARPE-19 cells, we measured the amount of VEGF secretion into the culture mediaum by ELISA. The ARPE-19 cells were treated with various concentrations of CAPE $(0,10$ or $20 \mu \mathrm{M})$ for $2 \mathrm{~h}$ prior to exposure to hypoxic conditions. Pre-treatment with various doses of CAPE led to a significant decrease in the production of VEGF, as measured in the cell supernatants $24 \mathrm{~h}$ following exposure to hypoxia (Fig. 2). As shown by the ELISA results, the VEGF levels were significantly increased in the ARPE-19 cells after $24 \mathrm{~h}$ of exposure to hypoxic conditions compared to normoxic conditions, and this increase was reversed by treatment with CAPE in a dose-dependent manner (Fig. 2).

Effects of CAPE on HIF-1 $\alpha$ activation under hypoxic conditions. Initially, in order to assess whether CAPE inhibits the HIF-1 $\alpha$ translocation, the ARPE-19 cells were incubated with $20 \mu \mathrm{M}$ CAPE under hypoxic conditions for $6 \mathrm{~h}$ (Fig. 3A). In the hypoxia-exposed ARPE-19 cells, the translocation of HIF-1 $\alpha$ to the nucleus was increased by almost 2-fold compared to the cells exposed to normoxic conditions. By contrast, pre-treatment of the cells with $20 \mu \mathrm{M}$ CAPE significantly inhibited the translocation of HIF-1 $\alpha$ under hypoxic conditions (Fig. 3A). Further experiments were carried out to determine whether the activation of HIF-1 $\alpha$ in the ARPE-19 cells is altered under hypoxic conditions. When nuclear extract proteins from the cells were probed with oligonucleotides within the VEGF promoter, subsequent gel shift analysis revealed a marked increase in HIF-1 $\alpha$ transcriptional activity in the ARPE-19 cells exposed to hypoxic conditions (Fig. 3B). However, the induction of specific HIF-1 $\alpha$ DNA binding activity by exposure to hypoxic conditions was inhibited by CAPE $(20 \mu \mathrm{M})$ (Fig. 3B). These results indicate that
A
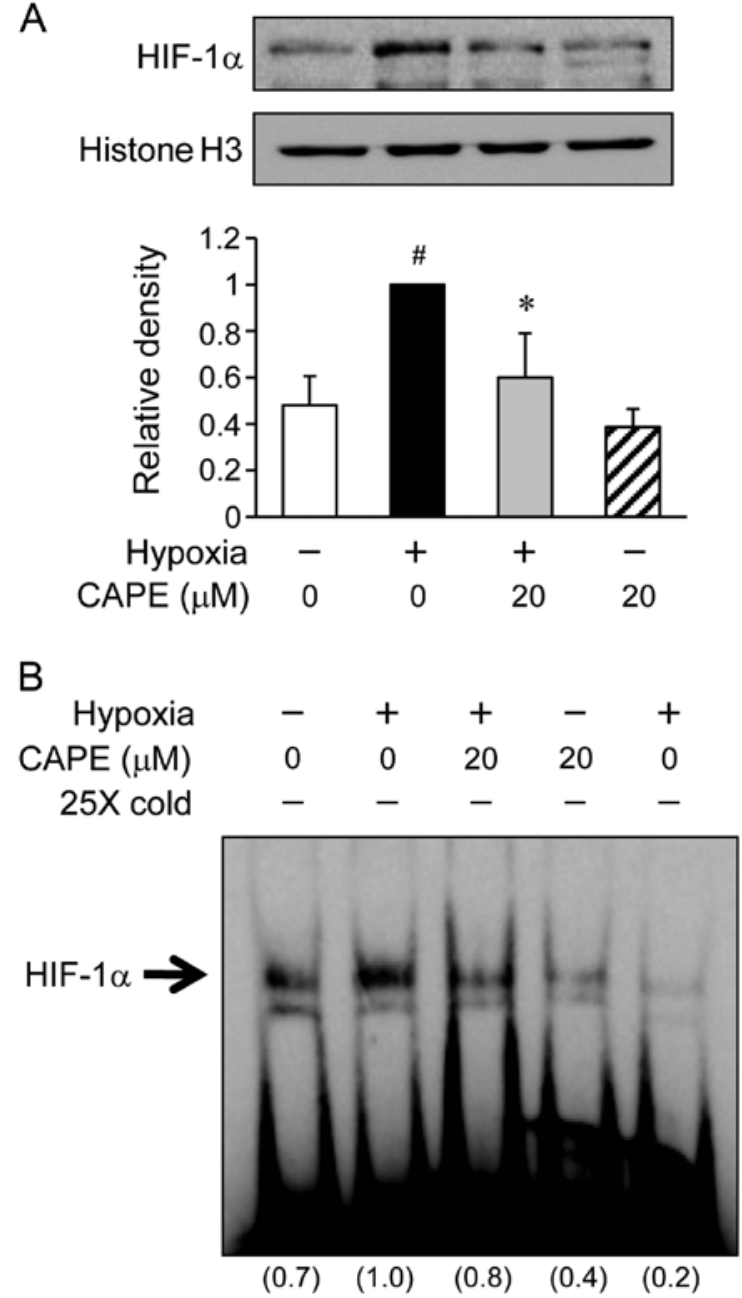

Figure 3. Inhibition of the activation of hypoxia-inducible factor-1 $\alpha$ (HIF-1 $\alpha)$ by caffeic acid phenethyl ester (CAPE) in ARPE-19 cells. (A) The HIF-1 $\alpha$ levels in the nuclear protein extracts were determined by western blot analysis. ARPE-19 cells were treated with CAPE $(20 \mu \mathrm{M}) 2 \mathrm{~h}$ prior to exposure to hypoxic conditions for $6 \mathrm{~h}$, and HIF- $1 \alpha$ was detected with its specific antibody. ${ }^{\#} \mathrm{p}<0.05$ vs. normoxia; " $\mathrm{p}<0.05$ vs. untreated group under hypoxic conditions. (B) Nuclear protein extracts $(5 \mu \mathrm{g})$ were prepared and analyzed for DNA binding activity of HIF-1 $\alpha$ using the electrophoretic mobility shift assay. ARPE-19 cells were treated with CAPE for $2 \mathrm{~h}$ prior to exposure to hypoxic conditions for $6 \mathrm{~h}$. The results shown are representative of 3 independent experiments. $25 \mathrm{X}$ cold indicates the molar fold excess of the specific competitor. To determine whether the observed shifted bands are specific for HIF-1 $\alpha$, competition tests are run: to a protein extract that displays intense shifted bands, additionally to the labeled HIF-1 $\alpha$ probe a non-labeled ('cold') oligonucleotide is added in excess. Cold competition oligonucleotides are used that either contain the wild type HIF-1 $\alpha$ recognition site. If the observed signals are HIF-1 $\alpha$-specific, those signals should disappear in the presence of the cold HIF-1 $\alpha$ wild-type competitor.

CAPE inhibits HIF-1 $\alpha$ activity by preventing the translocation of this transcription factor into the nucleus during hypoxia.

Effects of CAPE on the phosphorylation of phosphoinositide 3-kinase (PI3K)/AKT and mitogen-activated protein (MAP) kinases in hypoxia-exposed ARPE-19 cells. It is well known that the PI3K/AKT and MAP kinase signaling molecules are able to regulate HIF-1 $\alpha$ activation $(24,25)$. Therefore, we examined the effects of CAPE on the hypoxia-induced PI3K/AKT and MAP kinase activation. Western blot analysis revealed the accumulation of HIF-1 $\alpha$ at $6 \mathrm{~h}$ following exposure to hypoxic conditions (Fig. 4A). 


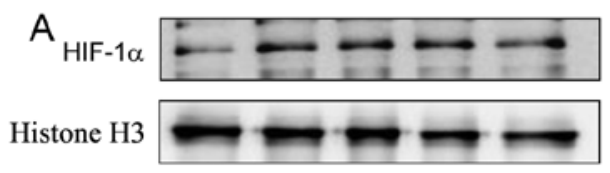

C
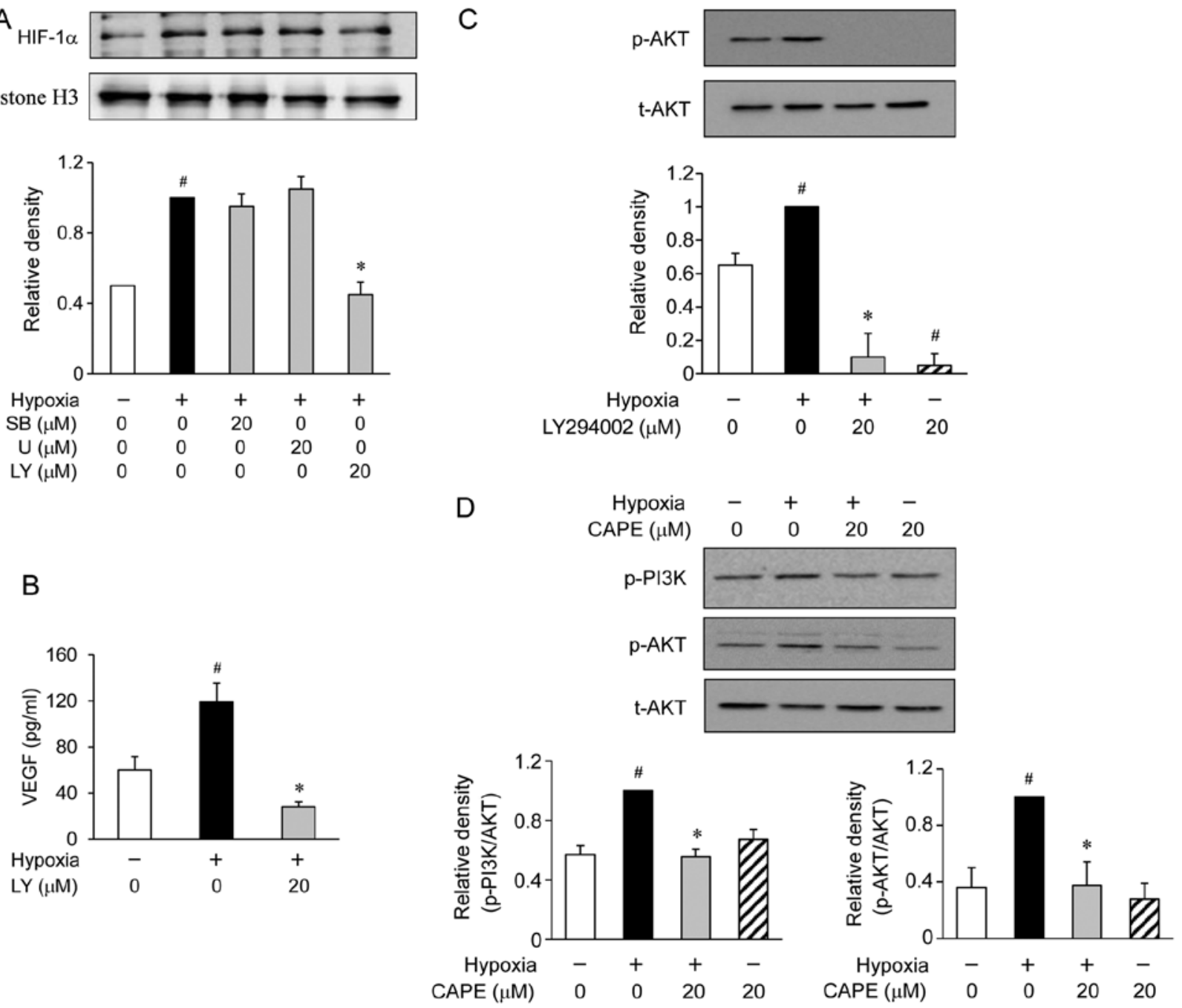

Figure 4. Effect of caffeic acid phenethyl ester (CAPE) on the phosphorylation of PI3K/AKT in ARPE-19 cells following exposure to hypoxia. (A) The levels of vascular endothelial growth factor (VEGF) in the culture medium were determined by ELISA. The hypoxia-inducible factor-1 $\alpha$ (HIF-1 $\alpha$ ) levels in the nuclear protein extracts were determined by western blot analysis. ARPE-19 cells were treated with SB203580 (SB; MAP kinase inhibitor), U0126 (U; ERK inhibitor) or LY294002 (LY; an inhibitor of PI3K/AKT) 5 min prior to exposure to hypoxia for $6 \mathrm{~h}$. (B) Cells were incubated with the indicated concentrations of LY294002 for $5 \mathrm{~min}$ prior to exposure to hypoxic conditions for $24 \mathrm{~h}$. The levels of VEGF were determined by ELISA. Each bar represents the mean \pm SD from 3 independent experiments. (C) Cells were incubated with the indicated concentrations of LY294002 for 5 min prior to exposure to hypoxic conditions for $24 \mathrm{~h}$. (D) Cells were incubated with CAPE $(20 \mu \mathrm{M}) 2 \mathrm{~h}$ prior to exposure to hypoxic conditions for $30 \mathrm{~min}$. Western blot analysis was performed for PI3K and AKT phosphorylation. ${ }^{*} \mathrm{p}<0.05$ vs. normoxia; ${ }^{*} \mathrm{p}<0.05$ vs. untreated group under hypoxic conditions.

When the ARPE-19 cells were treated with various inhibitors of signaling transduction pathways, such as SB203580 for p38 MAP kinase and U0126 for extracellular signal-regulated kinase (ERK), these inhibitors were not found to affect the accumulation of HIF-1 $\alpha$ during hypoxia (Fig. 4A). However, the ARPE-19 cells treated with LY294002 (an inhibitor of PI3K/AKT) showed a reduced accumulation of HIF-1 $\alpha$ induced by hypoxia (Fig. 4A). To determine whether the hypoxia-induced secretion of VEGF is associated with PI3K/ AKT, the ARPE-19 cells were exposed to hypoxia for $24 \mathrm{~h}$ in the presence or absence of $20 \mu \mathrm{M} \mathrm{LY} 294002$. We found that pre-treatment with LY294002 substantially reduced the hypoxia-induced production of VEGF (Fig. 4B). In addition, pre-treatment with LY294002 also reduced the phosphorylation of AKT (Fig. 4C). Based on these results, we investigated the effects of CAPE on the hypoxia-induced activation of PI3K/ AKT. The phosphorylation of AKT showed a marked increase within $2 \mathrm{~h}$ following exposure to hypoxic conditions. However, pre-treatment with CAPE resulted in a significant inhibition of the hypoxia-induced AKT phosphorylation (Fig. 4D). These results demonstrated that the inhibition of VEGF production by CAPE in the hypoxia-exposed ARPE-19 cells was associated with the downregulation of PI3K/AKT phosphorylation.

Effect of CAPE on hypoxia-induced intracellular ROS generation in ARPE-19 cells. In a previous study, ROS stimulated $\mathrm{CNV}$ by fostering a pro-angiogenic environment in the retina and choroid, and antioxidants were shown to reduce CNV (26). Therefore, in this study, we examined the inhibitory effects of CAPE on the hypoxia-induced generation of ROS (Fig. 5). Initially, in order to determine whether the hypoxia-induced production of VEGF is associated with ROS, the ARPE-19 cells were exposed to hypoxic conditions for $24 \mathrm{~h}$ in the presence or absence of ROS inhibitors, such as $10 \mu \mathrm{M}$ DPI for NADPH oxidase or $50 \mu \mathrm{M}$ YCG063 for mitochondrial ROS (Fig. 5A). These inhibitors significantly inhibited the 
A

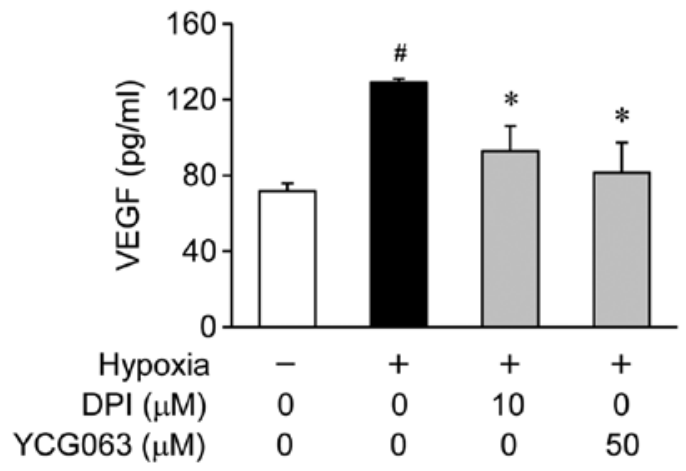

B
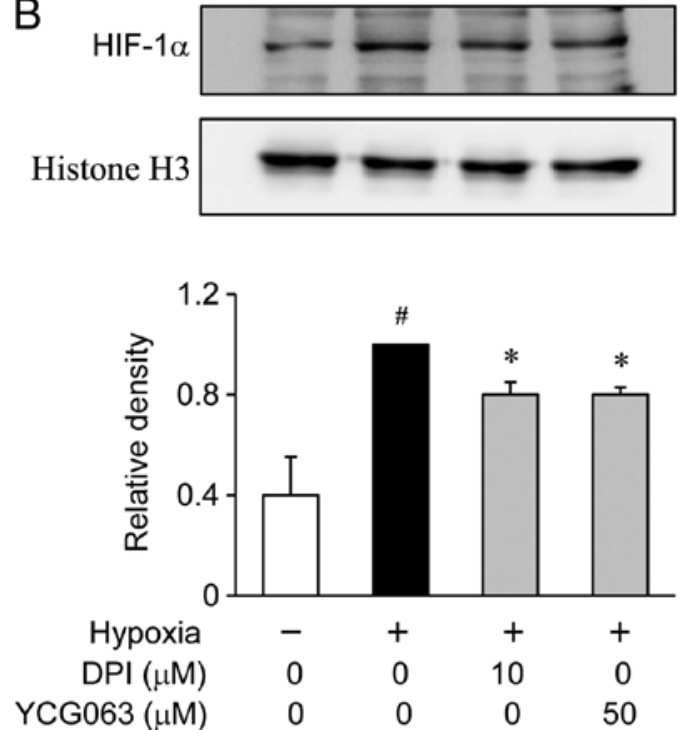

C
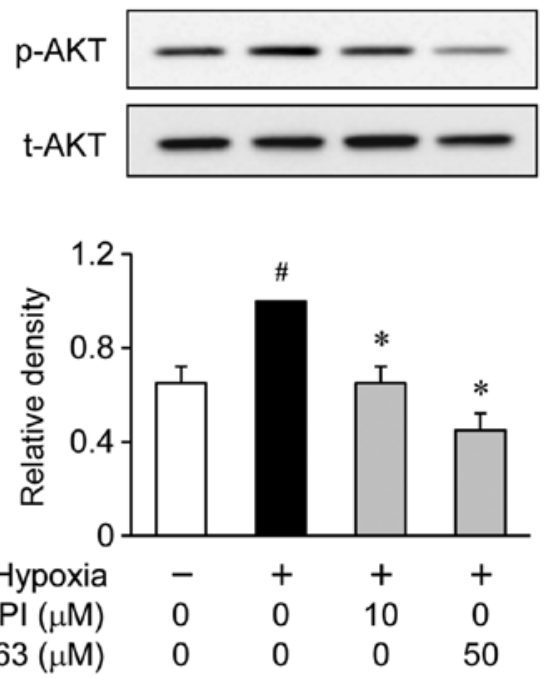

D

\footnotetext{
Figure 5. Effect of caffeic acid phenethyl ester (CAPE) on reactive oxygen species (ROS) production in ARPE-19 cells following exposure to hypoxic conditions. (A) The vascular endothelial growth factor (VEGF) levels were measured in the culture medium using a commercial ELISA kit. ARPE-19 cells were treated with diphenyleneiodonium (DPI) $(10 \mu \mathrm{M})$ or YCG063 (mitochondrial ROS inhibitor; $50 \mu \mathrm{M}) 5$ min prior to exposure to hypoxic conditions for $24 \mathrm{~h}$. (B) The hypoxia-inducible factor-1 $\alpha$ (HIF-1 $\alpha$ ) levels in the nuclear protein extracts were determined by western blot analysis. ARPE-19 cells were treated with DPI $(10 \mu \mathrm{M})$ or YCG063 $(50 \mu \mathrm{M}) 5$ min prior to exposure to hypoxic conditions for $6 \mathrm{~h}$, and HIF-1 $\alpha$ levels were detected with its specific antibody. (C) ARPE-19 cells were treated with DPI $(10 \mu \mathrm{M})$ or YCG063 $(50 \mu \mathrm{M}) 5 \mathrm{~min}$ prior to exposure to hypoxic conditions for $30 \mathrm{~min}$. Western blot analysis was performed for AKT phosphorylation. (D) Cells were incubated with CAPE $(20 \mu \mathrm{M}) 2 \mathrm{~h}$ prior to exposure to hypoxic conditions for $2 \mathrm{~h}$. ROS levels were measured by the DCF-DA assay. " $\mathrm{p}<0.05$ vs. normoxia; " $\mathrm{p}<0.05$ vs. untreated group under hypoxic conditions.
}

hypoxia-induced production of VEGF (Fig. 5A). Furthermore, when the ARPE-19 cells were treated with DPI and YCG063, these inhibitors reduced the accumulation of HIF-1 $\alpha$ in the nuclei during hypoxia (Fig. 5B). Since the production of VEGF in the hypoxia-exposed ARPE-19 cells was associated with the downregulation of AKT phosphorylation levels, we investigated whether AKT phosphorylation is associated with the generation of ROS. The ARPE-19 cells were exposed to hypoxic conditions for $2 \mathrm{~h}$ in the presence or absence of DPI and YCG063. The phosphorylation of AKT showed an increase within $2 \mathrm{~h}$ following exposure to hypoxic conditions. However, pre-treatment with DPI and YCG063 resulted in the significant attenuation of the hypoxia-induced AKT phosphorylation (Fig. 5C). These results demonstrate that the generation of ROS by exposure to hypoxic conditions serves as an upstream signal for the induction of VEGF production by PI3K/AKT activation. Therefore, we investigated whether pretreatment with CAPE inhibits the generation of ROS. We found that pre-treatment with CAPE markedly reduced the generation of ROS under hypoxic conditions (Fig. 5D).

\section{Discussion}

VEGF is known to be the most important modulator of both normal and pathological angiogenesis. The inhibition of VEGF function has been shown to lead to reduced pathological vessel formation in a murine model of ocular disease (27). RPE cells are one of the major cell constituents and secretors of VEGF 
in the retina (28). In the present study, in order to examine the association between hypoxia and angiogenesis, we measured the levels of VEGF production in human ARPE-19 cells using ELISA. The levels of VEGF were increased in a time-dependent manner. However, the maximum level of VEGF production was observed at the $24 \mathrm{~h}$ time point, and the levels subsequently decreased under hypoxic conditions compared to normoxic conditions in human ARPE-19 cells (Fig. 1). Treatment with concentrations of 10 and $20 \mu \mathrm{M}$ CAPE inhibited VEGF production under both normoxic and hypoxic conditions without inducing cytotoxicity.

It has been demonstrated that hypoxia induces the production of VEGF through the HIF transcriptional complex $(29,30)$. HIF comprises the $\alpha$ - and $\beta$-subunits, which heterodimerize to form a competent transcription factor (31). There are three isoforms of the $\alpha$-subunit, HIF- $1 \alpha$, HIF- $2 \alpha$ and HIF- $3 \alpha$, of which HIF-1 $\alpha$ is the most well characterized (32). HIF-1 $\alpha$ is constitutively expressed in the cytoplasm under normoxic conditions and is continually degraded. Under hypoxic conditions, HIF- $1 \alpha$ accumulates and is translocated to the nucleus, where it binds to the hypoxia response element of the VEGF promoter and induces transcriptional activity (31). HIF-1 $\alpha$ plays a pivotal role in angiogenesis. This suggests that hypoxia mediates CNV through HIF-1 $\alpha$-regulated VEGF production. Therefore, in this study, we investigated whether CAPE attenuates the translocation of HIF-1 $\alpha$ to the nucleus and its binding to hypoxia response element of the VEGF promoter. Our results demonstrated that CAPE reduces HIF-1 $\alpha$ accumulation (Fig. 3) and HIF-1 $\alpha$-dependent transcriptional activity (Fig. 3B). In previous studies, CAPE was shown to be associated with HIF stabilization under normoxic conditions $(33,34)$. In this study, however, CAPE reduced HIF-1 $\alpha$ accumulation. The reason for this discrepancy may lie in the different experimental conditions used (e.g., cell type, stimulus, species, etc.). In this regard, the inhibition of VEGF production by pre-treatment with CAPE may prove to be an effective therapeutic approach to relieve the progression of ocular angiogenesis through the regulation of HIF-1 $\alpha$.

In a previous study, the MAP kinase and PI3K/AKT signaling pathways were shown to be involved in regulating the expression of HIF-1 $\alpha$ and VEGF in laser-induced CNV in rats (35). Hypoxia activates the MAP kinases (36). The ERK and PI3K/AKT pathways have been known to be of critical importance for neovascularization, ischemia and angiogenesis $(37,38)$. It is possible that anti-neovascularization mechanisms are associated with the PI3K/AKT or MAP kinase pathways. To further elucidate the regulatory mechanisms of CAPE in these processes, we investigated whether the PI3K/AKT and MAP kinase signaling pathways are involved in regulating HIF-1 $\alpha /$ VEGF. Of note, p38 MAP kinase- and ERK-specific inhibitors did not suppress the expression of HIF-1 $\alpha$. However, the PI3Kspecific inhibitor completely inhibited the expression of HIF-1 $\alpha$. In addition, the PI3K-specific inhibitor significantly suppressed the expression of VEGF. Therefore, this finding strongly suggests that the PI3K/AKT signaling pathway, but not MAP kinases, plays a critical role in the expression of HIF-1 $\alpha$ /VEGF. Based on these results, we demonstrated a marked inhibition of the hypoxia-induced phosphorylation of AKT by CAPE in the ARPE-19 cells. Our findings suggest that the decrease in the hypoxia-induced production of VEGF by CAPE is due to the inhibition of HIF-1 $\alpha$ through the inactivation of the PI3K/AKT signaling pathway.

It has been reported that oxidative stress, which refers to the cellular damage caused by reactive oxygen intermediates (ROI), plays a causative role in both the initiation and progression of CNV and a contributing factor in AMD $(18,39)$. ROS also play a role in HIF-1 $\alpha$ induction (40). ROS are commonly produced during inflammatory processes, are involved in signal transduction and gene activation, and contribute to host cell and organ damage (41). ROS, including superoxide anion, hydroxyl radical and hydrogen peroxide, may play multiple roles in a number of diseases, such as atherosclerosis, angiogenesis, cancer, diabetes mellitus, neurological degeneration and asthma (42). N-acetylcysteine (NAC), a potent antioxidant, has been shown to inhibit the development of CNV in mice (43). It is well known that CAPE possesses significant antioxidant properties $(44,45)$. However, the effects of CAPE on hypoxia-induced ocular neovascularization and its molecular mechanisms have not yet been elucidated. In the present study, we demonstrated that ROS inhibitors significantly inhibited the activation of PI3K/AKT, as well as the expression of HIF- $1 \alpha$ and VEGF. Furthermore, we demonstrated that CAPE has intracellular ROS scavenging activity in ARPE-19 cells. Thus, the potential inhibition of ROS generation by CAPE is consistent with the inhibition of PI3K/AKT activation, HIF-1 $\alpha$ and VEGF expression and, thus, reduced ocular neovascularization.

In conclusion, the results obtained in the present study indicate that treatment of the ARPE-19 cells with CAPE decreases VEGF production following exposure to hypoxic conditions. CAPE significantly inhibited the accumulation of HIF-1 $\alpha$ under hypoxic conditions. The inhibitory effects of CAPE are mediated by the downregulation of PI3K/AKT activation and the inhibition of ROS signaling in ARPE-19 cells. These findings indicate that CAPE has the potential to target ROS, PI3K/AKT and HIF-1 $\alpha$ and inhibit VEGF production in ARPE-19 cells under hypoxic conditions. Such inhibitory effects may contribute to the treatment of various intraocular angiogenic diseases, such as AMD complicated by CNV, and may thus provide novel therapeutic efficacy.

\section{Acknowledgements}

This study was supported by a grant of the Korea Healthcare Technology R\&D Project, Ministry of Health and Welfare and Family Affairs, Republic of Korea (HI12C0005).

\section{References}

1. Oh JH, Oh J, Togloom A, Kim SW and Huh K: Effects of Ginkgo biloba extract on cultured human retinal pigment epithelial cells under chemical hypoxia. Curr Eye Res 38: 1072-1082, 2013.

2. Cao J, Zhao L, Li Y, et al: A subretinal matrigel rat choroidal neovascularization (CNV) model and inhibition of CNV and associated inflammation and fibrosis by VEGF trap. Invest Ophthalmol Vis Sci 51: 6009-6017, 2010.

3. Campochiaro PA: Ocular neovascularization. J Mol Med Berl 91: 311-321, 2013.

4. da Cruz L, Chen FK, Ahmado A, Greenwood J and Coffey P: RPE transplantation and its role in retinal disease. Prog Retin Eye Res 26: 598-635, 2007.

5. Jin J, Zhou KK, Park K, Hu Y, Xu X, Zheng Z, Tyagi P, Kompella UB and Ma JX: Anti-inflammatory and antiangiogenic effects of nanoparticle-mediated delivery of a natural angiogenic inhibitor. Invest Ophthalmol Vis Sci 52: 6230-6237, 2011. 
6. Vadlapatla RK, Vadlapudi AD and Mitra AK: Hypoxia-inducible factor-1 (HIF-1): A potential target for intervention in ocular neovascular diseases. Curr Drug Targets 14: 919-935, 2013.

7. Caprara C and Grimm C: From oxygen to erythropoietin: Relevance of hypoxia for retinal development, health and disease. Prog Retin Eye Res 31: 89-119, 2012.

8. Lutty G, Grunwald J, Majji AB, Uyama M and Yoneya S: Changes in choriocapillaris and retinal pigment epithelium in age-related macular degeneration. Mol Vis 5: 35-38, 1999.

9. Pouysségur J, Dayan F and Mazure NM: Hypoxia signalling in cancer and approaches to enforce tumour regression. Nature 441: 437-443, 2006

10. Vadlapatla RK, Vadlapudi AD, Pal D, Mukherji M and Mitra AK: Ritonavir inhibits HIF-1 $\alpha$-mediated VEGF expression in retinal pigment epithelial cells in vitro. Eye (Lond) 28: 93-101, 2014.

11. Moreira EF, Larrayoz IM, Lee JW and Rodríguez IR: 7-Ketocholesterol is present in lipid deposits in the primate retina: potential implication in the induction of VEGF and CNV formation. Invest Ophthalmol Vis Sci 50: 523-532, 2009.

12. Spilsbury K, Garrett KL, Shen WY, Constable IJ and Rakoczy PE: Overexpression of vascular endothelial growth factor (VEGF) in the retinal pigment epithelium leads to the development of choroidal neovascularization. Am J Pathol 157: 135-144, 2000

13. Kwak N, Okamoto N, Wood JM and Campochiaro PA: VEGF is major stimulator in model of choroidal neovascularization. Invest Ophthalmol Vis Sci 41: 3158-3164, 2000.

14. Krzystolik MG, Afshari MA, Adamis AP, Gaudreault J Gragoudas ES, Michaud NA, Li W, Connolly E, O'Neill CA and Miller JW: Prevention of experimental choroidal neovascularization with intravitreal anti-vascular endothelial growth factor antibody fragment. Arch Ophthalmol 120: 338-346, 2002.

15. Park SG, Lee DY, Seo SK, et al: Evaluation of anti-allergic properties of caffeic acid phenethyl ester in a murine model of systemic anaphylaxis. Toxicol Appl Pharmacol 226: 22-29, 2008

16. El-Refaei MF and El-Naa MM: Inhibitory effect of caffeic acid phenethyl ester on mice bearing tumor involving angiostatic and apoptotic activities. Chem Biol Interact 186: 152-156, 2010.

17. Jin UH, Song KH, Motomura M, Suzuki I, Gu YH, Kang YJ, Moon TC and Kim CH: Caffeic acid phenethyl ester induces mitochondria-mediated apoptosis in human myeloid leukemia U937 cells. Mol Cell Biochem 310: 43-48, 2008.

18. Beatty S, Koh H, Phil M, Henson D and Boulton M: The role of oxidative stress in the pathogenesis of age-related macular degeneration. Surv Ophthalmol 45: 115-134, 2000.

19. Izumi-Nagai K, Nagai N, Ohgami K, Satofuka S, Ozawa Y, Tsubota K, Ohno S, Oike Y and Ishida S: Inhibition of choroidal neovascularization with an anti-inflammatory carotenoid astaxanthin. Invest Ophthalmol Vis Sci 49: 1679-1685, 2008.

20. Apte RS, Richter J, Herndon J and Ferguson TA: Macrophages inhibit neovascularization in a murine model of age-related macular degeneration. PLoS Med 3: e310, 2006

21. Yu BC, Lee DS, Bae SM, et al: The effect of cilostazol on the expression of matrix metalloproteinase- 1 and type I procollagen in ultraviolet-irradiated human dermal fibroblasts. Life Sci 92: 282-288, 2013

22. Wang H and Joseph JA: Quantifying cellular oxidative stress by dichlorofluorescein assay using microplate reader. Free Radic Biol Med 27: 612-616, 1999.

23. Uehara H, Luo L, Simonis J, Singh N, Taylor EW and Ambati BK Anti-SPARC oligopeptide inhibits laser-induced CNV in mice. Vision Res 50: 674-679, 2010.

24. Semenza G: Signal transduction to hypoxia-inducible factor 1. Biochem Pharmacol 64: 993-998, 2002.

25. Minet E, Michel G, Mottet D, Raes M and Michiels C: Transduction pathways involved in hypoxia-inducible factor-1 phosphorylation and activation. Free Radic Biol Med 31: 847-855, 2001.

26. LeeHS, Jun JH,Jung EH,Koo BA and Kim YS: Epigalloccatechin3-gallate inhibits ocular neovascularization and vascular permeability in human retinal pigment epithelial and human retinal microvascular endothelial cells via suppression of MMP-9 and VEGF activation. Molecules 19: 12150-12172, 2014.

27. Adamis AP and Shima DT: The role of vascular endothelial growth factor in ocular health and disease. Retina 25: 111-118, 2005 .
28. Forooghian F, Razavi R and Timms L: Hypoxia-inducible factor expression in human RPE cells. Br J Ophthalmol 91: 1406-1410, 2007.

29. Ratcliffe PJ, O'Rourke JF, Maxwell PH and Pugh CW: Oxygen sensing, hypoxia-inducible factor- 1 and the regulation of mammalian gene expression. J Exp Biol 201: 1153-1162, 1998

30. Ratcliffe PJ, Pugh CW and Maxwell PH: Targeting tumors through the HIF system. Nat Med 6: 1315-1316, 2000.

31. Semenza GL: HIF-1: Mediator of physiological and pathophysiological responses to hypoxia. J Appl Physiol (1985) 88: 1474-1480, 2000.

32. Sparkenbaugh EM, Ganey PE and Roth RA: Hypoxia sensitization of hepatocytes to neutrophil elastase-mediated cell death depends on MAPKs and HIF-1 $\alpha$. Am J Physiol Gastrointest Liver Physiol 302: G748-G757, 2012.

33. Liu Y, Zhang B, Zhang J, Wang S, Yao H, He L, Chen L, Yue W, Li Y and Pei X: CAPE promotes the expansion of human umbilical cord blood-derived hematopoietic stem and progenitor cells in vitro. Sci China Life Sci 57: 188-194, 2014.

34. Roos TU, Heiss EH, Schwaiberger AV, Schachner D, Sroka IM, Oberan T, Vollmar AM and Dirsch VM: Caffeic acid phenethyl ester inhibits PDGF-induced proliferation of vascular smooth muscle cells via activation of p38 MAPK, HIF-1 $\alpha$, and heme oxygenase-1. J Nat Prod 74: 352-356, 2011.

35. Yang XM, Wang YS, Zhang J, Li Y, Xu JF, Zhu J, Zhao W, Chu DK and Wiedemann P: Role of PI3K/Akt and MEK/ERK in mediating hypoxia-induced expression of HIF-1alpha and VEGF in laser-induced rat choroidal neovascularization. Invest Ophthalmol Vis Sci 50: 1873-1879, 2009.

36. Seta KA, Spicer Z, Yuan Y, Lu G and Millhorn DE: Responding to hypoxia: Lessons from a model cell line. Sci STKE 2002: re11, 2002.

37. Bullard LE, Qi X and Penn JS: Role for extracellular signal-responsive kinase-1 and -2 in retinal angiogenesis. Invest Ophthalmol Vis Sci 44: 1722-1731, 2003.

38. Ackah E, Yu J, Zoellner S, et al: Akt1/protein kinase Balpha is critical for ischemic and VEGF-mediated angiogenesis. J Clin Invest 115: 2119-2127, 2005.

39. Li X, Cai Y, Wang YS, Shi YY, Hou W, Xu CS, Wang HY, Ye Z, Yao LB and Zhang J: Hyperglycaemia exacerbates choroidal neovascularisation in mice via the oxidative stress-induced activation of STAT3 signalling in RPE cells. PLOS One 7: e47600, 2012.

40. Chandel NS, McClintock DS, Feliciano CE, Wood TM, Melendez JA, Rodriguez AM and Schumacker PT: Reactive oxygen species generated at mitochondrial complex III stabilize hypoxia-inducible factor-1alpha during hypoxia: A mechanism of $\mathrm{O} 2$ sensing. J Biol Chem 275: 25130-25138, 2000.

41. Jung WK, Heo SJ, Jeon YJ, Lee CM, Park YM, Byun HG, Choi YH, Park SG and Choi IW: Inhibitory effects and molecular mechanism of dieckol isolated from marine brown alga on COX-2 and iNOS in microglial cells. J Agric Food Chem 57: 4439-4446, 2009.

42. Yang D, Elner SG, Bian ZM, Till GO, Petty HR and Elner VM: Pro-inflammatory cytokines increase reactive oxygen species through mitochondria and NADPH oxidase in cultured RPE cells. Exp Eye Res 85: 462-472, 2007.

43. Hara R, Inomata Y, Kawaji T, Sagara N, Inatani M, Fukushima M and Tanihara H: Suppression of choroidal neovascularization by $\mathrm{N}$-acetyl-cysteine in mice. Curr Eye Res 35: 1012-1020, 2010

44. Bai H, Liu R, Chen HL, Zhang W, Wang X, Zhang XD, Li WL and Hai CX: Enhanced antioxidant effect of caffeic acid phenethyl ester and Trolox in combination against radiation induced-oxidative stress. Chem Biol Interact 207: 7-15, 2014.

45. Ozguner F, Altinbas A, Ozaydin M, Dogan A, Vural H, Kisioglu AN, Cesur G and Yildirim NG: Mobile phone-induced myocardial oxidative stress: Protection by a novel antioxidant agent caffeic acid phenethyl ester. Toxicol Ind Health 21: 223-230, 2005. 